\title{
Shock wave versus iontophoresis in patients with carpal tunnel syndrome
}

\author{
DOI: https://doi.org/10.5114/pq.2020.96420
}

\author{
Reda Kotb Abd Elrazik', Hanan Hosny M. Battesha², Sara Mohamed Samir ${ }^{3}$ \\ ${ }^{1}$ Department of Physical Therapy for Musculoskeletal Disorders and Its Surgery, Faculty of Physical Therapy, \\ Benha University, Benha, Egypt \\ ${ }^{2}$ Department of Physical Therapy for Neuromuscular Disorders and Its Surgery, Faculty of Physical Therapy, \\ Modern University for Technology and Information, Cairo, Egypt \\ ${ }^{3}$ Department of Physical Therapy for Musculoskeletal Disorders and Its Surgery, Faculty of Physical Therapy, \\ Cairo University, Cairo, Egypt
}

\section{Abstract}

Introduction. Carpal tunnel syndrome (CTS) is the most commonly encountered nerve entrapment disorder; it causes pain, numbness, tingling sensation in the hands and leads to work disability. The purpose of this study was to analyse the impact of shock wave and iontophoresis in patients with CTS.

Methods. This study was conducted in 30 patients with CTS of both sexes, aged 30-50 years. The patients were randomly allocated into 2 groups. Study group A received shock wave therapy and study group B received iontophoresis therapy.

Results. The results revealed a higher improvement in group A than in group B. In group A, there were a significant increase of sensory nerve conduction velocity of median nerve and a significant decrease of pain after treatment, while in group $B$, there were a less significant increase of sensory nerve conduction and less significant reduction of pain after treatment. The inter-group comparisons showed statistically significant differences in favour of group A.

Conclusions. Shock wave is more effective than iontophoresis in the treatment of patients with CTS.

Key words: shock wave, iontophoresis, nerve conduction velocity, pain, carpal tunnel syndrome

\section{Introduction}

Carpal tunnel syndrome (CTS) is characterized by a pressure of the median nerve in the wrist. It is the most common compressive issue and its most consistent explanation is idiopathic. Even though relapsing is possible, pain and paraesthesia in the thumb, first two fingers, and the lateral portion of the ring finger are the most well-known clinical highlights of CTS. Paraesthesia and tactile deficiencies may include the whole palm except the thenar eminence [1].

The disorder has received much attention in recent years as it might be connected with occupations that require utilization of the hands, e.g., computer users or painters. Numerous individuals present this condition regardless of the work they do [2].

Anatomically, there are two destinations of median nerve pressure: one at the degree of the proximal furthest reaches of the carpal passage, brought about by wrist flexion as a result of changes in thickness and solidness of the lower arm fascia and the proximal part of the flexor retinaculum; and the other at the degree of the tightest part, near the hamate bone. Nerve pressure may consecutively lead to issues identifying with intra-neural blood microcirculation, injuries at the degree of the myelin sheath and axonal level, and changes to the supporting connective tissue [3].

Nerve conduction velocity (NCV) is always evaluated to assess and analyse illnesses of the peripheral nerves. These investigations explicitly measure the conduction speed, latency, and amplitude just as the state of the reaction follow- ing electrical stimulation of the nerve through the skin and the tissues [4].

It has previously been established that sensory nerve conduction studies are the most sensitive electrodiagnostic tests to confirm the diagnosis of CTS. Sensory nerve conduction studies are important in the documentation of sensory fibre involvement in CTS. Nerve conduction studies are the only electrophysiological means to confirm the clinically defined diagnosis of CTS [5].

Extracorporeal shock wave treatment (ESWT) reduced manifestations of CTS as local steroid infusion. Shockwaves can instigate a critical increase of nitric oxide and a decline in pro-inflammatory substances, e.g., nuclear factor-kappa $\mathrm{B}(\mathrm{NF}-\kappa \mathrm{B})$, as well as incite neovascularization and improve blood supply and tissue recovery [6].

lontophoresis is a non-invasive modality which utilizes electric flow to convey a charged or neutral particle through cell membrane. It builds the entrance of ionized medication. The procedure uses an equivalent limited quantity of current for the conveyance of medication. The particles that are conveyed by the assistance of the iontophoresis technique have some charge on them: either cationic or anionic. Inactive recovery corticosteroids are the prime sedate. They are applied because they have a significant calming impact, less successful, and can be administered both orally and topically. Different corticosteroids are accessible as water solvent salts, having a negative charge and thus moving towards the separate anode within the sight of electrical current. Dexamethasone with lidocaine is utilized to treat musculoskeletal is-

Correspondence address: Reda Kotb Abd Elrazik Gad Elhak, Department of Physical Therapy for Musculoskeletal Disorders and Its Surgery, Faculty of Physical Therapy, Benha University, Fareed Nada Street, Benha, Qalubiya Governorate, Arab Republic of Egypt, e-mail: Dr_reda_kotb@yahoo.com 
sues, where the medication is kept at the positive terminal as it is a negative charge particle and it is moved to the skin [7].

The specific objective of this study was to analyse the impacts of shock wave and iontophoresis in patients with CTS.

\section{Subjects and methods}

\section{Design}

Two-factorial pre- and post-study design was used in the randomized study with intra-rater reliability and inter-rater agreement.

\section{Sampling}

A total of 30 patients of both sexes with CTS were willing to take part in this investigation. They were chosen from the Outpatient Clinics of Neurology and Orthopaedics at the Faculty of Physical Therapy, Modern University for Technology and Information, Cairo, between December 2018 and April 2019. All patients with CTS suffered from pain, numbness, and/or tingling sensation in the lateral three and a half fingers. Anonymity and privacy were guaranteed and each method was performed in consistence with the applicable laws and institutional rules. The age, weight, height, and body mass index of the patients were established. The participants were randomized into 2 groups by using a roll of a dice: study group $\mathrm{A}(n=15)$ took the odd numbers, while study group $B$ $(n=15)$ took the even numbers. The groups were treated with shock wave therapy and iontophoresis therapy to compare their effect on CTS.

Subjects eligible to participate in this study were those with CTS but we excluded 10 patients owing to their postoperative status, recent upper limb injury, or hand deformities. All patients included in this study had CTS diagnosed by a neurologist or orthopaedist and confirmed by a CT scan; all were clinically diagnosed with hand thenar discomfort pain, as well as positive Phalen's test and Tinel's test.

The exclusion criteria involved peripheral neuropathy, carpal bone fractures, joint injuries, and/or cervical spondylosis with sensory radiculopathy.

\section{Assessment}

Before the evaluation commencement, the participants' height and weight were evaluated and body mass index calculated.

NCV was used to measure sensory conduction velocity of median nerve before and after the treatment program in both groups. The assessment device consists of a display screen, electrode junction box, integrated operation panel, mouse, keyboard, printer, power unit, and stimulator.

Visual analogue scale (VAS) was applied before and after the treatment program in both groups to evaluate pain intensity by marking a 100-mm line with 2 extremes. VAS is a unidimensional measure of pain intensity, which has been widely used in diverse adult populations; the simplest VAS is a straight horizontal line of fixed length, usually $100 \mathrm{~mm}$. Its ends represent the worst (left side) and the lightest (right side) detected level of pain.

\section{Treatment}

The shock wave device consists of a body and head that introduce shock waves and a screen. The instrument was routinely calibrated by the manufacturer and it was calibrated before starting the study. Shock waves cause micro-trauma and hematoma formation, which eventually leads to osteoblastic activity, increased callus formation, and bone healing, as well as relieves pain due to insertional tendinopathy by provoking a painful level of hyper-stimulation analgesia.

Iontophoresis machines work by conveying little, safe electronic ions to the skin. A direct (galvanic) electrical flow is led through the electrode with dexamethasone and lidocaine. In this way, ions are applied to the skin, causing a response that definitely diminishes pain, irritation, and paraesthesia.

\section{Evaluation procedures}

This was a median sensory conduction study, antidromic, with patients in the supine position or sitting with arm extended beside the body. Recording electrodes acted as active electrodes: a ring electrode was placed on mid-portion of the proximal phalanx of the index finger (or centre finger). As for the reference electrode, a ring electrode was placed on the mid-portion of the middle phalanx of the index finger (or centre finger). The ground electrode was placed between the stimulating and recording electrodes. The stimulation electrode (anode is $2.5 \mathrm{~cm}$ proximal to cathode) was applied 12-14 cm proximally to the active recording electrode.

The measure of pain that a patient feels extends over a continuum from none to an outrageous measure of pain. An 11-point scale (ranging from 0 to 10) was applied, with 0 standing for 'no pain,' 1-2 for 'little pain,' 3-6 for 'moderate pain,' 7-9 for 'extreme pain,' and 10 for 'the most sever pain'. VAS has good validity and test-retest reliability of $0.95-0.97$.

\section{Treatment procedures}

Patients in group A (11 females and 4 males) received a program of shock wave therapy of 2000 pulses per session at an energy flux density of $0.03 \mathrm{~mJ} / \mathrm{mm}^{2}, 1.6$ bars per session, in the area between the thenar and hypothenar eminences of the hand, for about 5 minutes for each session, 3 sessions per week for 6 weeks.

Patients in group B (10 females and 5 males) received iontophoresis treatment with dexamethasone and lidocaine delivered to the tissues at the palmar lower surface of the forearm (at the area of the median nerve passage under the carpal tunnel) of the affected hand. The delivered substance should be of ionic nature and put under the anode with a comparative charge (i.e. negatively charged ions placed under the negative electrode or cathode and positively charged ions placed under the anode). The anode applies the positively charged substance into the skin. The cathode delivers the negatively charged substance to the skin. The electrode with an ionic substance is known as the active, treatment, or delivery electrode. The other one is used to complete the circuit and is called the inactive, indifferent, or return electrode. Particles with the same polarity are repelled into the skin. The treatment duration was 20 minutes per session, 3 sessions per week, for 6 weeks.

\section{Allocation}

Overall, 40 patients were screened for eligibility and only 30 satisfied the inclusion criteria and completed the investigation. They were randomly assigned into 2 groups ( $A$ and $B$ ). Before the study, all participants received full, detailed information about the trial design. Figure 1 shows the flow chart of the study. 


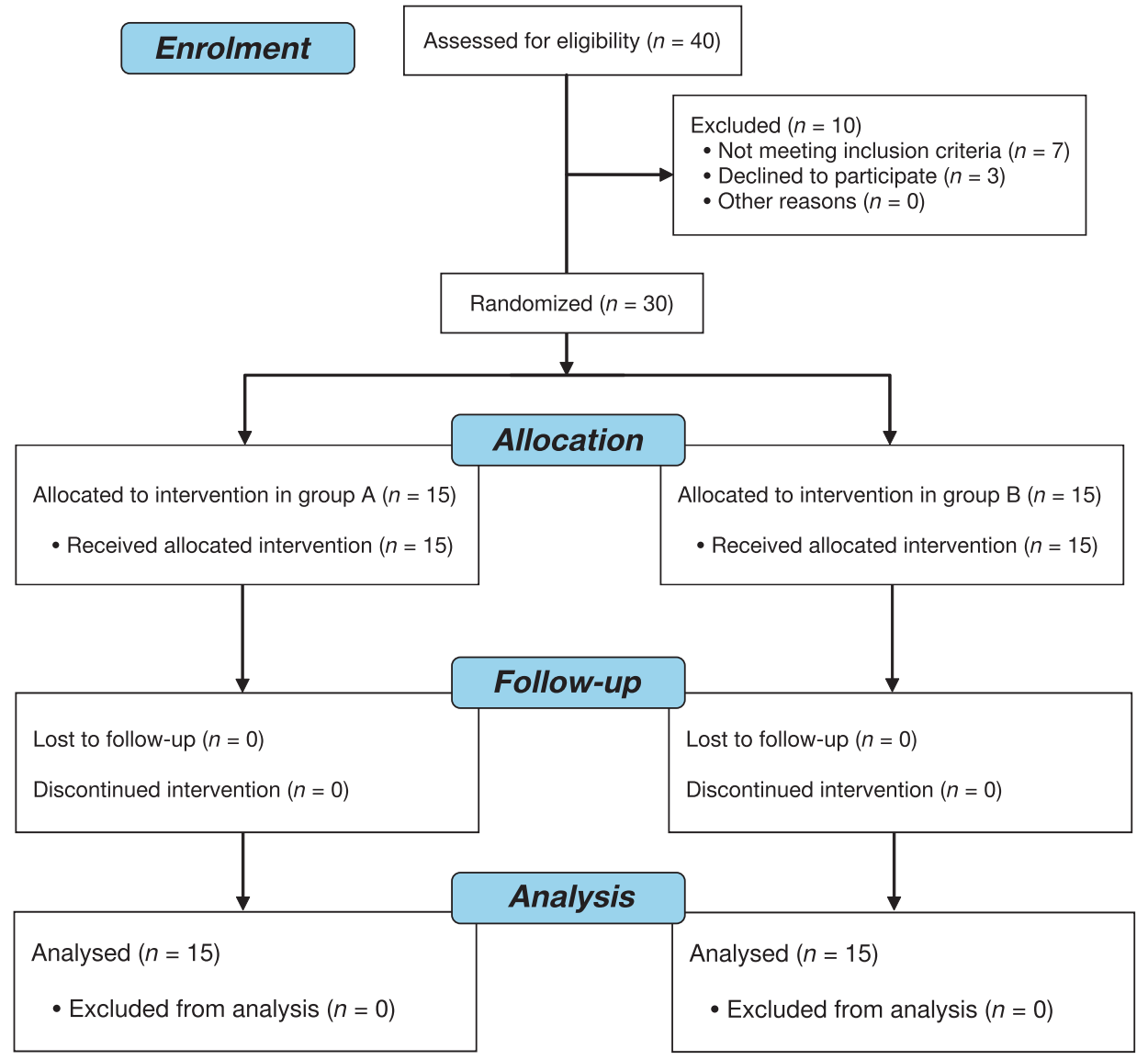

Figure 1. The study flow chart

\section{Statistical analysis}

Statistical analysis was conducted by using the SPSS software for Windows, version 25 (SPSS, Inc., Chicago, USA). The study involved 2 dependent variables: VAS score and sensory median NCV (SNCV). Before the final analysis, data were screened for normality assumption with the ShapiroWilk $W$ test, which showed that the data were normally distributed for SNCV and not normally distributed for VAS score after treatment. Parametric test, paired sample tests and independent sample $t$-tests, were applied for inferential analysis of the SNCV dependent variable, while the Wilcoxon signed ranks and Mann-Whitney tests were used for within-group and between-group comparison of results. The initial alpha level was 0.05 for all tests.

To avoid a type II error, a preliminary power analysis [power $(1-\alpha$ error $p)=0.95, \alpha=0.05$, effect size $=1.435]$ determined a sample size of 14 for each group in the study. This effect size was calculated in accordance with a pilot study performed among 12 participants (6 in each group), considering the distribution of plantar pressure under the centre of the right heel as a primary outcome. The power analysis was carried out by the $G^{*}$ Power 3.1.9.2 software, with the use of a test family as $t$-test and a statistical test as mean difference between 2 independent groups.

\section{Ethical approval}

The research related to human use has complied with all the relevant national regulations and institutional policies, has followed the tenets of the Declaration of Helsinki, and has been approved by the Institutional Ethics Committee at the Faculty of Physical Therapy, Cairo University, Egypt (No. P.T. REC/012/002727).

\section{Informed consent}

Informed consent has been obtained from all individuals included in this study.

\section{Results}

\section{General demographic data}

No statistically significant differences $(p>0.05)$ were observed between the subjects in both groups with regard to general characteristics (Table 1).

Table 1. Comparison of baseline demographic data between the study groups

\begin{tabular}{|c|c|c|c|c|}
\hline \multirow{2}{*}{ Characteristics } & \multicolumn{2}{|c|}{ Before treatment } & \multirow{2}{*}{$p$} & \multirow{2}{*}{ Significance } \\
\hline & Group A $(n=15)$ & Group B $(n=15)$ & & \\
\hline Age (years) & $30.06 \pm 2.86$ & $30.6 \pm 4.37$ & 0.67 & Not significant \\
\hline Weight (kg) & $76.53 \pm 1.68$ & $75.6 \pm 2.38$ & 0.36 & Not significant \\
\hline Height (cm) & $175.53 \pm 1.68$ & $174.33 \pm 5.48$ & 0.69 & Not significant \\
\hline Body mass index $\left(\mathrm{kg} / \mathrm{m}^{2}\right)$ & $24.84 \pm 0.71$ & $24.92 \pm 1.38$ & 0.8 & Not significant \\
\hline
\end{tabular}


Comparison of SNCV and VAS score: before vs. after treatment, within and between groups

The pre-treatment comparison of VAS and SNCV variables between the groups also showed no significant difference, with the $Z$ value of -1.073 for VAS and mean difference of -0.569 (Table 2). Moreover, the post-test comparison between the groups revealed a statistically significant difference regarding the VAS score $(Z$ value $=-3.256)$ and SNCV (95\% Cl: $-7.041,-4.706)$ in favour of group A (Table 2). The within-group comparison demonstrated a significant improvement (decrease) in the VAS score in both groups, with the percentage of improvement equal $88 \%$ and $61 \%$ in groups $A$ and B, respectively (Table 2 and Figure 2). Also, NCV statistically significantly increased in both groups, with the percent- age of improvement equal $18 \%$ and $8 \%$ in groups $A$ and $B$, respectively (Table 2 and Figure 3 ).

\section{Discussion}

This study demonstrated a more significant improvement in SNCV in group A than in group $B$, as well as a more significant reduction of pain in group $A$ than in group $B$ as a result of the influence of shock wave being more effective than iontophoresis in patients with CTS.

These results are in agreement with a study by Atthakomol et al. [8], who analysed viability in diminishing pain and improving clinical capacity between single-dose ESWT and local corticosteroid infusions over 12 weeks. There was a fundamentally more prominent improvement in manifestations,

Table 2. Mean \pm SD of VAS score and SNCV; comparison before vs. after treatment, within and between groups

\begin{tabular}{|l|c|c|c|c|c|c|}
\cline { 2 - 3 } \multicolumn{1}{c|}{} & \multicolumn{2}{c|}{$p^{*}$} & \multicolumn{2}{c|}{ SNCV (median) } & \multicolumn{2}{c|}{$p$} \\
\cline { 2 - 3 } \cline { 5 - 7 } & Before & After & & Before & After & \\
\hline Group A $(n=15)$ & $(6) 5.933 \pm 1.387$ & $(1) 0.733 \pm 0.458$ & $0.001^{* * *}$ & $41.71 \pm 1.56$ & $50.647 \pm 1.99$ & 0.001 \\
\hline Group B $(n=15)$ & $(5) 5.267 \pm 1.981$ & (2) $2.067 \pm 1.335$ & $0.001^{* * *}$ & $41.035 \pm 0.995$ & $44.77 \pm 0.96$ & 0.001 \\
\hline$p^{* *}$ & $0.305^{* * *}$ & $0.002^{* * *}$ & & $0.166^{* * *}$ & $<0.001^{* * *}$ & $<$ \\
\hline
\end{tabular}

VAS - visual analogue scale, SNCV - sensory median nerve conduction velocity

* comparison between pre- and post-treatment values within each group

** between-group comparison

${ }^{* * *}$ significant $p$ value $(<0.05)$
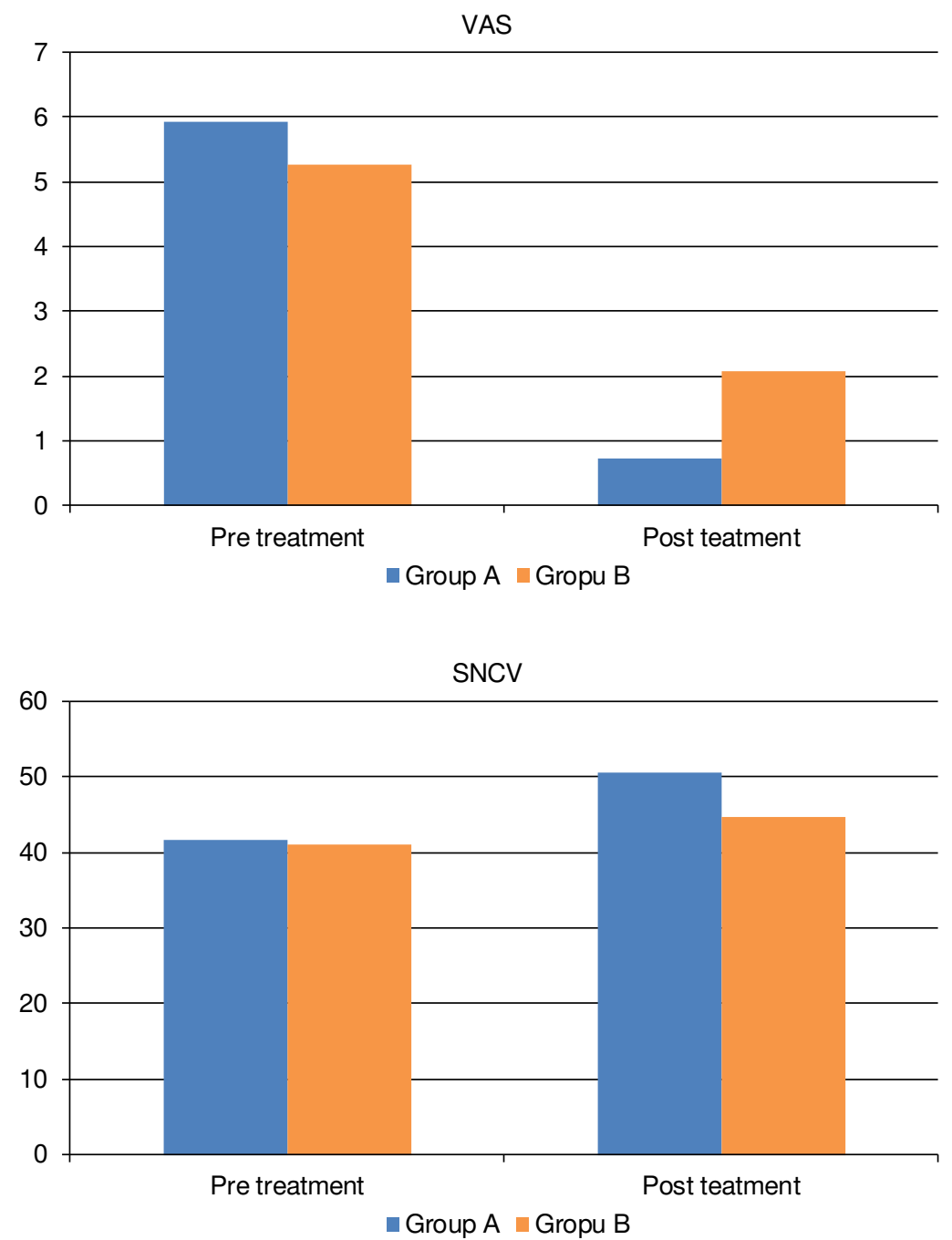

Figure 2. Mean visual analogue scale (VAS) scores before and after treatment in both groups
Figure 3. The results of sensory nerve conduction velocity (SNCV) of median nerve before and after treatment in both groups 
functional level, and Boston questionnaire scores in the ESWT group as compared with the local corticosteroid group. A critical decrease of pain was observed in the ESWT group, while the local corticosteroid group presented no significant decline of pain in a similar period of treatment.

A study by Chen et al. [9] was in line with our study and indicated that shock waves caused micro-trauma and hematoma arrangement, eventually leading to osteoblastic activity, expanded callus development, and bone healing. Shock wave treatment alleviated pain due to insertional tendinopathy by provoking hyper-analgesia. The modality demonstrated $56-90 \%$ efficacy rates in the treatment of soft tissue disorders, including calcifying tendinitis of the shoulder, plantar fasciitis, and tennis elbow.

A previous study by Rompe et al. [10] compared the outcomes achieved in 15 patients with painful disorders treated with 1000 impulses of shock waves at $0.06 \mathrm{MPa}$ given multiple times ( 3 times) weekly with the results among an equal number of patients treated with placebo. There was a significant alleviation of pain and improvement of function in the shock wave treated individuals. ESWT reduced pain by overactivation of the nerves carrying pain sensations to the cerebrum. The strategy can separate excessive calcification stores; it treats the condition that caused the spur, not the spur itself.

The results of the current study were consistent with those achieved by Daeschler et al. [11], who imply that the underlying mechanisms of ESWT enhanced angiogenesis, growth factor synthesis, and modulation of the inflammatory response. For nerve regeneration, shock wave therapy was reported to increase the proliferation rate and expression of regenerative phenotype-associated markers like glial fibrillary acidic protein and c-Jun in Schwann cells.

Moreover, Weiland et al. [12] found that shock wave therapy had an effect in patients with chronic conditions who did not respond to traditional therapy. ESWT quickens the body's own healing process through cavitation, and stimulates fibroblast and tenocyte generation. Fibroblast or tenocyte cells make up connective tissues as ligaments and the fascia.

The current study is supported by several studies [13-20] which indicate that VAS score is helpful to detect the level of pain in patients with CTS. Physiotherapy research on rehabilitation of CTS allows to develop more effective conservative treatments and increases the chance to avoid or delay surgical intervention. Immediately introduced simple anti-inflammatory therapy by electrical current may bring a quick and permanent recovery but patients suffer from several symptoms such as burning pain lasting for a few days.

In line with the present findings, Torro et al. [21], utilized the transdermal organization of corticosteroids by means of iontophoresis, which involved restricted entrance, with a considerable number of issues treated with systemic administration. Dexamethasone infiltration into human tissues is also encouraged, but at small, limited areas. It can be used in the management of several musculoskeletal and neuromuscular disorders, such as muscle irritation, CTS, and osteoarthritis of the knee; all these conditions could be treated by iontophoresis but need long-duration therapy (12-24 weeks) to show results.

The results of this study contradict with the findings achieved by Anderson et al. [22], who suggest that corticosteroids are exceptionally powerful in diminishing irritation and particle exchange to chloride particles that the ionic current carriers. The aftereffects of human trials propose that the current magnitude and duration should be considered as factors in treating musculoskeletal dysfunctions with ionto- phoresis by using dexamethasone. This improvement was attributed to working with long-duration treatment to produce effects.

\section{Limitations}

The authors are aware of the study limitations. The examination was constrained by diminished patient's capacity to complete the assessment and treatment procedures, as the patients suddenly felt headache, pain, blurred vision, and fatigue (powerless and tired inclination).

\section{Conclusion}

In view of the findings of this study, it was inferred that shock wave was more effective than iontophoresis in patients with CTS.

\section{Acknowledgments}

The authors are grateful to Prof. Dr. Naguib Salem, Dean of the Faculty of Physical Therapy, Modern University for Technology and Information, who permitted to perform all examinations and treatment procedures in the laboratories and medical centre of the Faculty of Physical Therapy. Finally, we cannot forget to thank all the patients and colleagues who participated in this research for their cooperation throughout the study.

\section{Disclosure statement}

No author has any financial interest or received any financial benefit from this research.

\section{Conflict of interest}

The authors state no conflict of interest.

\section{References}

1. Kohara N. Clinical and electrophysiological findings in carpal tunnel syndrome [in Japanese]. Brain Nerve. 2007;59(11):1229-1238.

2. Stevens JC, Witt JC, Smith BE, Weaver AL. The frequency of carpal tunnel syndrome in computer users at a medical facility. Neurology. 2001;56(11):1568-1570; doi: 10.1212/wnl.56.11.1568.

3. Lundborg G. Nerve injury and repair. London: Churchill Livingstone; 2008.

4. Aramideh M, Ongerboer de Visser BW. Brainstem reflexes: electrodiagnostic techniques, physiology, normative data, and clinical applications. Muscle Nerve. 2002;26(1): 14-30; doi: 10.1002/mus.10120.

5. Kasius KM, Claes F, Verhagen WIM, Meulstee J. Motor nerve conduction tests in carpal tunnel syndrome. Front Neurol. 2019;10:149; doi: 10.3389/fneur.2019.00149.

6. Hems T. Peripheral nerve injuries and repair. In: Bentley $\mathrm{G}$ (ed.), European surgical orthopaedics and traumatology. Berlin-Heidelberg: Springer; 2014; 1555-1577.

7. Sharma S, Parvez N, Sharma PK. lontophoresis-models and applications: a review. African J Basis Appl Sci. 2015;7(1):1-7; doi: 10.5829/idosi.ajbas.2015.7.1.9236.

8. Atthakomol P, Manosroi W, Phanphaisarn A, Phrompaet S, lammatavee S, Tongprasert S. Comparison of single-dose radial extracorporeal shock wave and local corticosteroid injection for treatment of carpal tunnel syndrome including mid-term efficacy: a prospective randomized controlled trial. BMC Musculoskelet Disord. 2018;19(1):32; doi: 10.1186/s12891-018-1948-3. 
9. Chen Y-J, Wang C-J, Yang KD, Kuo Y-R, Huang H-C, Huang Y-T. Extracorporeal shock waves promote healing of collagenase-induced Achilles tendinitis and increase TGF-beta1 and IGF-I expression. J Orthop Res. 2004;22(4):854-861; doi:10.1016/j.orthres.2003.10.013.

10. Rompe JD, Zoellner J, Nafe B. Shock wave therapy versus conventional surgery in the treatment of calcifying tendinitis of the shoulder. Clin Orthop Relat Res. 2001; 387:72-82; doi: 10.1097/00003086-200106000-00010.

11. Daeschler SC, Harhaus L, Schoenle P, Boecker A, Kneser U, Bergmeister KD. Ultrasound and shock-wave stimulation to promote axonal regeneration following nerve surgery: a systematic review and meta-analysis of preclinical studies. Sci Rep. 2018;8(1):3168; doi: 10.1038/ s41598-018-21540-5.

12. Weiland D, Lee $C$, Ugarte R, Monga M. Impact of shockwave coupling on efficacy of extracorporeal shockwave lithotripsy. J Endourol. 2007;21(2):137-140; doi: 10.1089/ end.2006.0179.

13. Łabuzek M, Grochowska A, Mika M, Wojtaszek K. Subjective assessment of post-operative pain in patients hospitalised in surgical wards. Medical Studies. 2019;35(2): 100-107; doi: 10.5114/ms.2019.86328.

14. Rzadkiewicz MM, Wójtowicz S, Rozenek H. Screening preoperative anxiety - when does it relate to the quality of life among patients before total joint replacement? Preliminary reports. Health Psychol Rep. 2019;7(2):139-148; doi: 10.5114/hpr.2019.85660.

15. Rezaei S, Hassanzadeh S. Are mindfulness skills associated with reducing kinesiophobia, pain severity, pain catastrophizing and physical disability? Results of Iranian patients with chronic musculoskeletal pain. Health Psychol Rep. 2019;7(4):276-285; doi: 10.5114/hpr.2019. 84747.

16. De Siqueira SRDT, Teixeira JM. Sensory interaction theory: revision of the craniofacial region. Adv Psychiatry Neurol. 2019;28(1):34-41; doi: 10.5114/ppn.2019.84358.

17. Hamoda RE, Osman DA, Hamada HA, Radwan R, Yousef AM, Samea GAA, et al. Effect of myofascial release on electrophysiological and clinical measures of pregnant women with carpal tunnel syndrome. Physiother Quart. 2019;27(1):18-24; doi: 10.5114/pq.2019.83057.

18. Zych-Litwin C, Litwin JA. Complex regional pain syndrome: diagnosis and treatment at the very onset as the key to success? A case report with implications for first contact doctors. Reumatologia. 2019;57(2):117-119; doi: 10.5114/reum.2019.84818.

19. Bobowik PŻ. Effectiveness of physiotherapy in carpal tunnel syndrome (CTS). Adv Rehabil. 2019;33(2):47-58; doi: 10.5114/areh.2019.85023.

20. Turczyn P, Kosińska B, Janikowska-Hołoweńko D, Malec-Milewska M, Marszalec N, Maleszka P, et al. Translation and cross-cultural adaptation of the Polish Central Sensitization Inventory. Reumatologia. 2019;57(3):129134; doi: 10.5114/reum.2019.86422.

21. Torro J, Brunetti L, Patel DK. Iontophoretic administration of dexamethasone for musculoskeletal pain. J Musculoskel Med. 2011;28(11):410-421.

22. Anderson CR, Morris RL, Boeh SD, Panus PC, Sembrowich WL. Effects of iontophoresis current magnitude and duration on dexamethasone deposition and localized drug retention. Phys Ther. 2003;83(2):161-170; doi: 10.1093/ptj/83.2.161. 